\title{
Satisfacción del profesorado con el Grado en Administración de Empresas Teacher satisfaction with the Degree in Business Administration
}

\author{
Miguel Angel Acedo Ramírez, Francisco Javier Ruiz Cabestre \\ miguel-angel.acedo@unirioja.es, javier.ruiz@unirioja.es \\ Departamento de Economía y Empresa \\ Universidad de La Rioja \\ Logroño, España
}

\begin{abstract}
Resumen- El objetivo del presente trabajo es evaluar la satisfacción de los profesores del Grado en Administración y Dirección de Empresas de la Universidad de La Rioja durante el curso académico 2015-16. Los resultados indican que el nivel de satisfacción es adecuado, salvo en lo que se refiere a la dedicación del estudiante a sus estudios. Otros aspectos que tienen que ver con el alumno que requieren cierta mejora son los conocimientos previos que traen los estudiantes y su asistencia a clase. También parece necesario mejorar la asignación de horarios entre los profesores y el plan de formación del profesorado. Finalmente, destaca la alta valoración de las prácticas realizadas por los estudiantes en las empresas.
\end{abstract}

Palabras clave: satisfacción, educación superior, Grado en Administración y Dirección de Empresas

Abstract- The objective of this paper is to evaluate the satisfaction of the professors of the Degree in Business Administration and Management of the University of La Rioja during the academic year 2015-16. The results indicate that the level of satisfaction is adequate except in what concerns with the dedication of the students to the Degree. Other aspects that have to do with the student that require some improvement are the previous knowledge of the students and their attendance to classes. The allocation of timetables of teachers and the teacher-training plan also require improvement. Finally, highlight the high valuation of the internships carried out by students in companies.

Keywords: satisfaction, higher education, Degree in Business Administration

\section{INTRODUCCIÓN}

El Espacio Europeo de Educación Superior (EEES) ha supuesto un cambio importante en el sistema universitario español desde su implantación en el curso académico 2009/10, cuyo objetivo es la mejora de la calidad de la enseñanza universitaria (Ariza et al., 2014). Las cambios que se han producido en el sistema universitario pretenden la mejora y modernización, a través de la excelencia, la internacionalización y la dimensión social de la Educación Superior (Ministerio de Educación, 2010). La integración del sistema universitario español en el EEES, tal y como se recoge en la Declaración de Bolonia y en el título XIII de la Ley Orgánica de Universidades (BOE 307, 24 de diciembre de 2001), supone cambios en el papel del alumno -como centro de atención- y el profesor -como tutor y asesor-, en el sistema de créditos y en la propia estructura de los estudios, entre otros. Todos estos cambios persiguen una formación universitaria de calidad y, por ende, una mayor satisfacción de todos los agentes implicados (Ariza et al., 2013). Aunque las ideas básicas del nuevo sistema son atractivas y sugerentes, han surgido en su aplicación a la realidad dificultades que pueden haber condicionado la consecución de los objetivos previstos con su implantación (Otero et al., 2012).

Para alcanzar estos objetivos, la calidad y satisfacción se vuelven elementos importantes dentro del sistema universitario. Se han realizado numerosos trabajos, donde se analizan diferentes cuestiones relacionadas con el EEES y la enseñanza universitaria ${ }^{1}$, pero hay menos sobre las dimensiones que configuran la satisfacción con los Grados de los distintos usuarios (Alves y Raposo, 2007). Algunos trabajos han analizado la satisfacción de los estudiantes de grado (Alves y Raposo, 2007), mientras otros han analiza la satisfacción de los egresados con los estudios de Máster (González y Sanchís, 2014). También existen trabajos sobre la satisfacción del profesorado antes del proceso de convergencia europea (Ferrer i Julià, 2004; Cardona et al., 2009), sin embargo, un estudio actualizado una vez implementado el EEES parece más que necesario.

El profesor es uno de los principales agentes del proceso educativo, por lo que la evaluación de su satisfacción es fundamental para la universidad. El nuevo marco de educación superior, dado que busca un mayor compromiso del profesorado con la universidad en su labor docente, investigadora y de extensión universitaria, no tendría, en principio, por qué contribuir a incrementar su satisfacción. En la medida en que el nuevo modelo contribuya a una mayor satisfacción del profesorado, tendremos un beneficio para la universidad en la que desempeña sus funciones, aumentando

${ }^{1}$ El aprendizaje por competencias (Cáceres-Lorenzo y SalasPascual, 2012; Ion y Cano, 2012; Mateo y Vlachopoulos, 2013), la mejora del proceso de enseñanza-aprendizaje (SaizManzanares y Payo-Hernández, 2012), la importancia de la evaluación formativa (Fraile et al., 2013); la creación de las Escuelas de Doctorado (Castro et al., 2012) y la calidad del servicio (Capelleras y Veciana, 2001). 
su prestigio (Anaya y Suárez, 2007). No obstante, todo cambio implica un rechazo y el profesor universitario no es una excepción, por lo que quizás la implementación del modelo no se esté desarrollando conforme a lo planificado (Monereo, 2010a; 2010b).

El presente trabajo analiza la satisfacción de los profesores con el programa formativo del Grado en Administración y Dirección de Empresas (GADE), impartido en la Facultad de Ciencias Empresariales de la Universidad de La Rioja (UR) desde el curso académico 2009/10, dado que el conocimiento de los distintos aspectos que influyen en la satisfacción, es relevante para diseñar e implementar acciones de mejora en dicho Grado.

El trabajo se estructura de la siguiente forma: en el segundo apartado se pone de manifiesto la importancia, en el sistema universitario, de tener profesores satisfechos y se describe el marco en el que se desarrolla el Grado en ADE; en el tercero se detalla la base de datos utilizada; en el cuarto se muestran los resultados obtenidos y, para finalizar, en el quinto apartado, se recogen las principales conclusiones.

\section{CONTEXTO}

La reforma de las enseñanzas universitarias oficiales en España, a través de la Ley Orgánica 4/2007 de 12 de abril, ha potenciado la autonomía de las universidades y aumentado la exigencia de rendir cuentas sobre el cumplimiento de sus funciones. Ello ha propiciado el incremento de las expectativas y exigencias de los distintos usuarios del sistema universitario respecto al papel que debe desempeñar (Capelleras y Veciana, 2001). La universidad se entiende como un servicio público, por lo que "las políticas públicas deberán reconocer las diversas misiones de la educación superior, que van desde la docencia y la investigación al servicio a la comunidad, y la implicación en la cohesión social y el desarrollo cultural” (Comisión Europea, 2009).

El número de universitarios no ha dejado de crecer $\mathrm{y}$, sin embargo, el tamaño de su mercado potencial se ha reducido, debido a la disminución de la tasa de natalidad, a la vez que han aparecido nuevas alternativas de formación y se ha incrementado el número de universidades privadas. Además, se ha producido un aumento de la movilidad de los estudiantes y una gradual internacionalización del sistema universitario (Lozano, 2003; Marcet, 2001; Bengoetxea y Buela-Casal, 2013), fomentado por el nuevo marco europeo que facilita la movilidad de alumnos y profesores, así como el reconocimiento de estudios entre los países (Quevedo et al., 2015). El proceso de internacionalización de los procesos educativos se extiende más allá de las fronteras de Europa, generando una competencia entre universidades a nivel global (Bengoetxea y Buela-Casal, 2013).

Todos estos hechos han contribuido a que las universidades se preocupen tanto por la calidad del servicio que prestan como por la satisfacción de los distintos grupos de interés, como son los estudiantes, el profesorado, el personal de administración y servicios, los potenciales empleadores y la sociedad en general (Álvarez y Rodríguez, 1997).

Con respecto a la calidad, cabe señalar que una de las principales innovaciones de la Ley Orgánica 6/2001, de 21 de diciembre, de universidades se refiere a la introducción de mecanismos externos de evaluación de la calidad universitaria conforme a objetivos y procedimientos transparentes, para lo que crea la Agencia Nacional de Evaluación de la Calidad y Acreditación (ANECA). Esto ha propiciado que las universidades hayan desarrollado Sistemas de Garantía Interna de Calidad (SGIC) ante la necesidad de acreditación, dado que los incentivos de evaluar la calidad han estado centrados en la acreditación (Abreu, 2015).

En relación a la satisfacción, en el caso de la Universidad de La Rioja, el SGIC de los planes de estudio recoge procedimientos de análisis y seguimiento de los distintos colectivos implicados. En concreto, la evaluación de la satisfacción del profesorado y del alumnado. Centrándonos en la satisfacción del profesorado, cabe señalar que es prioritario para la universidad tener un profesorado satisfecho, dado que es uno de los agentes más importantes del proceso educativo y ello redundará en su propio beneficio (Anaya y Suárez, 2007). Sin embargo, parece existir un cierto rechazo al cambio entre el profesorado universitario, por lo que no está claro su implicación en el nuevo modelo derivado del Espacio Europeo (Monereo, 2010a). El coste de cambio a los nuevos métodos de enseñanza y aprendizaje puede explicar dicha resistencia al cambio (Monereo, 2010b). Otros trabajo, en cambio, son más favorables y proponen una aceptación del cambio por parte del profesor universitario y una actitud positiva al nuevo modelo (Fernández et al., 2010). Por ello, y a pesar de que hay estudios que analizan la opinión de los profesores en relación con la calidad y satisfacción con la educación superior antes de la implantación del Espacio Europeo (Ferrer i Julià, 2004; Cardona et al., 2009), una valoración del nuevo modelo se torna más que necesaria, ante la falta de estudios en este ámbito.

La satisfacción de los profesores con los estudios que están impartiendo es un concepto subjetivo, porque depende de sus expectativas y deseos (Athiyama, 1997), y multifacético, porque su análisis se ve condicionado por los distintos elementos que influyen en ella, así como los diferentes enfoques para su medición (Pérez et al., 2010). La satisfacción debe incluir aspectos que el profesorado considere relevantes para valorar el proceso de forma global (Oldfield y Baron, 2000). Aspectos no solo relacionados con el proceso de enseñanza y el plan de estudios, sino también con otras cuestiones relacionados con su organización, recursos humanos, etc. (Firdaus, 2006).

Por tanto, el objetivo de este trabajo es analizar la satisfacción de los profesores con determinados aspectos relacionados con los nuevos grados (el alumnado, la organización y desarrollo de la enseñanza, y los recursos humanos y materiales), derivados del Espacio Europeo de Educación Superior y, más concretamente, con el Grado en Administración y Dirección de Empresas, impartido en la Facultad de Ciencias Empresariales de la Universidad de La Rioja.

Desde el curso académico 2019/10 se viene impartiendo el Grado en ADE en la Universidad de La Rioja, con una oferta de plazas de nuevo de ingreso de 150 alumnos. La enseñanza es de carácter presencial y se imparte en castellano, a excepción de varias asignaturas para las que hay posibilidad de cursarlas en inglés. El grado se estructura en cuatro cursos: en los tres primeros se imparten asignaturas comunes y de carácter obligatorio, y en el cuarto curso se ofertan asignaturas de carácter optativo (conducentes a la obtención de las menciones de Dirección y Organización de Empresas; 
Contabilidad y Finanzas; Planificación y Gestión de Marketing, y Derecho Económico de la Empresa). Con independencia del itinerario elegido el estudiante debe cursar un total de 240 créditos ECTS, de los cuales 180 se corresponden con asignaturas básicas y obligatorias, 36 con optativas, 12 con prácticas externas y 12 con el trabajo fin de grado. Tanto el trabajo fin de grado como las prácticas son de carácter obligatorio y se realizan en cuarto curso.

Para evaluar la satisfacción de los profesores se ha utilizado el cuestionario sobre "Satisfacción del profesorado de Grado con el programa formativo”, que forma parte del Sistema de Garantía Interna de Calidad (SGIC) de la Universidad de la Rioja y es uno de los indicadores que la Agencia Nacional de Evaluación de la Calidad y la Acreditación (ANECA) tiene en cuenta para la renovación de la acreditación de los títulos de Grado. A través de esta encuesta se evalúan los distintos aspectos relacionados con la actividad del profesorado. En particular, se analizan las siguientes dimensiones:

\section{A. Alumnado}

En este apartado se analizan cuestiones como la satisfacción con los conocimientos previos de los alumnos, la dedicación de estos a sus estudios, la asistencia a clase, la adquisición de competencias, los resultados académicos y la percepción del profesorado sobre la satisfacción de los alumnos con el Grado.

\section{B. Organización y desarrollo de la enseñanza}

En este campo se abordan temas como la organización del plan de estudios, los programas de acogida, el contenido de las guías docentes, las metodologías de enseñanza-aprendizaje, la coordinación horizontal y vertical, la asignación de créditos a materias, los horarios, las prácticas, la movilidad, las tutorías y los criterios de evaluación.

\section{Recursos humanos y materiales}

Se analizarán cuestiones como la satisfacción del profesorado con el sistema de garantía de la calidad, el plan de formación, la cualificación y experiencia del personal de administración y servicios (Biblioteca, Oficina del Estudiante, Secretarías de Decanato, Conserjería, etc.) y del profesorado, las instalaciones e infraestructuras, los recursos tecnológicos, los recursos para la docencia, las acciones de mejora docente, la información publicada en la web del título, el sistema de sugerencias y quejas, el Equipo Directivo del Centro y las materias impartidas.

\section{DESCRIPCIÓN}

Para la realización del estudio se recogió información de los profesores que han impartido clase en el Grado en ADE durante el curso académico 2015/2016, a través de un cuestionario que consta de 32 preguntas que se agrupan en las tres áreas o dimensiones indicadas en el apartado anterior. Cada uno de los ítems se mide en una escala Likert de 1 a 10 , donde el 1 significa “mínimo grado de satisfacción” y el 10 sería “máximo grado de satisfacción”. En la tabla 1 se detalla la ficha técnica del estudio. Para que puedan emitir juicios relativos a los distintos aspectos que se evalúan a través del cuestionario, la encuesta se realiza cuando el curso académico estaba avanzado. En concreto, se efectuó a finales del segundo semestre, de tal forma que los profesores pudieran tener una opinión formada sobre el programa formativo.
Tabla 1. Ficha técnica del estudio sobre la satisfacción del profesorado con GADE

\begin{tabular}{l|l}
\hline Denominación & $\begin{array}{l}\text { Satisfacción del profesorado con el } \\
\text { Grado en ADE }\end{array}$ \\
\hline Universo & $\begin{array}{l}75 \text { profesores de GADE del curso } \\
\text { 2015/16 en la Universidad de La Rioja }\end{array}$ \\
\hline Unidad de análisis & $\begin{array}{l}\text { Profesores de GADE que han contestado } \\
\text { el cuestionario al finalizar el curso } \\
\text { académico 2015/16 }\end{array}$ \\
\hline $\begin{array}{l}\text { Técnica de } \\
\text { recogida de datos }\end{array}$ & $\begin{array}{l}\text { Cuestionario estructurado contestado on } \\
\text { line por los profesores }\end{array}$ \\
\hline $\begin{array}{l}\text { Tamaño de la } \\
\text { muestra }\end{array}$ & 34 encuestas válidas \\
\hline Error muestral & $\pm 12,51 \%$ 95\% (P=Q=0,5) \\
\hline $\begin{array}{l}\text { Fecha del trabajo } \\
\text { de campo }\end{array}$ & $\begin{array}{l}\text { Segundo semestre del curso académico } \\
2015 / 16\end{array}$ \\
\hline $\begin{array}{l}\text { Técnicas de } \\
\text { análisis empleadas }\end{array}$ & Análisis descriptivo e inferencial \\
\hline
\end{tabular}

Los profesores que contestaron la encuesta al final del curso académico de forma voluntaria y anónima fueron 34, lo que representa una tasa de respuesta del $45,3 \%$.

\section{RESUltados}

Los resultados se exponen siguiendo los tres bloques en los que se estructura el cuestionario: (1) alumnado; (2) organización y desarrollo de la enseñanza, y (3) recursos humanos y materiales.

\section{A. Alumnado}

De los resultados obtenidos (ver tabla 2), destaca la percepción sobre la satisfacción de los alumnos con el programa formativo (7,12 puntos), en nuestro caso, Grado en Administración y Dirección de Empresas, seguido de la satisfacción con las competencias adquiridas por los estudiantes $(6,97)$ y sus resultados académicos $(6,65)$, todos ellos con valores significativamente mayores a 6 . El profesorado expresa un nivel de satisfacción menor, pero con valores significativamente mayores a 5 , sobre los conocimientos previos de los estudiantes $(5,44)$ y su asistencia a clase $(5,62)$. Finalmente, el aspecto peor valorado es el que se refiere a la dedicación de los estudiantes a lo planificado en la titulación $(5,44)$, que no es significativamente mayor que 5.

Tabla 2. Estadísticos descriptivos sobre el alumnado

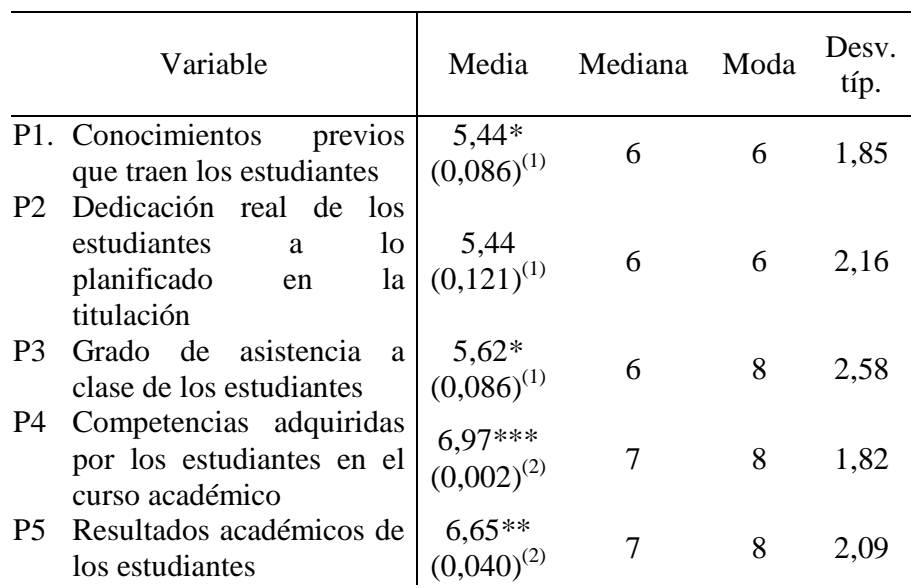


P6 Percepción sobre la

satisfacción de los $7,12 * * *$

\begin{tabular}{l|llll} 
alumnos con el programa & $(0,000)^{(2)}$ & 7,5 & 8 & 1,75
\end{tabular}

formativo

Nota: (1) La hipótesis nula es que el valor de media es menor o igual a 5, frente a la hipótesis alternativa de que es mayor a 5. (2) La hipótesis nula es que el valor de media es menor o igual a 6 , frente a la hipótesis alternativa de que es mayor a 6 . Los p-valor de la $t$ student, que permiten contrastar dichas hipótesis, se recogen entre paréntesis. *** Significativo al $1 \%$. ** Significativo al $5 \%$. * Significativo al $10 \%$.

Fuente: Elaboración propia

\section{B. Organización y desarrollo de la enseñanza}

En el bloque relativo al plan de estudios y estructura (ver tabla 3), la cuestión mejor valorada por los profesores se refieren al desarrollo de las prácticas externas curriculares (8,32 puntos), con un valor significativamente superior a 8 , aunque también las extracurriculares o voluntarias obtienen una muy buena valoración $(8,24)$.

Tabla 3. Estadísticos descriptivos sobre la organización y desarrollo de la enseñanza

\begin{tabular}{|c|c|c|c|c|c|}
\hline \multicolumn{2}{|r|}{ Variable } & Media & Mediana & Moda & Desv. \\
\hline P7 & $\begin{array}{l}\text { Organización del plan } \\
\text { de estudios }\end{array}$ & $\begin{array}{l}7,26 * * * \\
(0,001)^{(2)}\end{array}$ & 8 & 9 & 2,15 \\
\hline P8 & Programas de acogida & & & & \\
\hline & $\begin{array}{l}\text { y orientación al } \\
\text { alumno de nuevo } \\
\text { ingreso }\end{array}$ & $\begin{array}{c}7,68 * * \\
(0,035)^{(3)}\end{array}$ & 8,5 & 9 & 2,11 \\
\hline P9 & $\begin{array}{l}\text { Contenido de las guías } \\
\text { docentes }\end{array}$ & $\begin{array}{l}8,00 * * * \\
(0,003)^{(3)}\end{array}$ & 8,5 & 9 & 1,95 \\
\hline P10 & $\begin{array}{l}\text { Metodologías de } \\
\text { enseñanza- } \\
\text { aprendizaje } \\
\text { en el utilizadas } \\
\text { formativo }\end{array}$ & $\begin{array}{c}7,62 * * \\
(0,040)^{(3)}\end{array}$ & 8 & 8 & 2,00 \\
\hline P11 & $\begin{array}{l}\text { Coordinación } \\
\text { secuenciación } \\
\text { horizontal de las } \\
\text { materias }\end{array}$ & $\begin{array}{c}7,65 * * \\
(0,036)^{(3)}\end{array}$ & 8 & 8 & 2,03 \\
\hline P12 & $\begin{array}{l}\text { Coordinación y } \\
\text { secuenciación vertical } \\
\text { de las materias }\end{array}$ & $\begin{array}{c}7,62 * * \\
(0,042)^{(3)}\end{array}$ & 8 & 8 & 2,02 \\
\hline P13 & $\begin{array}{l}\text { Asignación de } \\
\text { créditos a las distintas } \\
\text { materias }\end{array}$ & $\begin{array}{l}7,32 * * * \\
(0,000)^{(2)}\end{array}$ & 8 & 8 & 2,08 \\
\hline P14 & $\begin{array}{l}\text { Procedimiento de } \\
\text { asignación de horarios }\end{array}$ & $\begin{array}{c}6,21^{* *} \\
(0,013)^{(1)}\end{array}$ & 7 & 7 & 3,03 \\
\hline P15 & $\begin{array}{l}\text { Adecuación de los } \\
\text { complementos de } \\
\text { formación para } \\
\text { facilitar el aprendizaje } \\
\text { en el título }\end{array}$ & $\begin{array}{c}7,74^{* *} \\
(0,013)^{(3)}\end{array}$ & 8 & 9 & 1,85 \\
\hline P16 & $\begin{array}{l}\text { Desarrollo de las } \\
\text { prácticas externas - } \\
\text { curriculares- de los } \\
\text { alumnos }\end{array}$ & $\begin{array}{c}8,32 * \\
(0,091)^{(4)}\end{array}$ & 9 & 9 & 1,39 \\
\hline P17 & $\begin{array}{l}\text { Desarrollo de las } \\
\text { prácticas externas - } \\
\text { voluntarias- de los } \\
\text { alumnos }\end{array}$ & $\begin{array}{l}8,24 * * * \\
(0,000)^{(3)}\end{array}$ & 9 & 9 & 1,60 \\
\hline P18 & $\begin{array}{llr}\text { Programas } & & \text { de } \\
\text { movilidad de } & \text { los }\end{array}$ & $\begin{array}{l}7,26 * * * \\
(0,001)^{(2)}\end{array}$ & 7 & 7 & 2,09 \\
\hline
\end{tabular}

\begin{tabular}{|c|c|c|c|c|c|}
\hline & estudiantes & & & & \\
\hline P19 & $\begin{array}{l}\text { Satisfacción con las } \\
\text { tutorías }\end{array}$ & $\begin{array}{l}7,85^{* * *} \\
(0,008)^{(3)}\end{array}$ & 8 & 8 & 1,97 \\
\hline P20 & $\begin{array}{l}\text { Procedimientos } \mathrm{y} \\
\text { criterios de evaluación }\end{array}$ & $7,53^{*}$ & & & \\
\hline & $\begin{array}{l}\text { utilizados en la } \\
\text { titulación }\end{array}$ & $(0,068)^{(3)}$ & 8 & 8 & 2,02 \\
\hline
\end{tabular}

Nota: (1) La hipótesis nula es que el valor de media es menor o igual a 5, frente a la hipótesis alternativa de que es mayor a 5. (2) La hipótesis nula es que el valor de media es menor o igual a 6 , frente a la hipótesis alternativa de que es mayor a 6. (3) La hipótesis nula es que el valor de media es menor o igual a 7, frente a la hipótesis alternativa de que es mayor a 7. (4) La hipótesis nula es que el valor de media es menor o igual a 8, frente a la hipótesis alternativa de que es mayor a 8. Los p-valor de la t-student, que permite contrastar dichas hipótesis, se recogen entre paréntesis. *** Significativo al $1 \%$. ** Significativo al 5\%. * Significativo al 10\%.

Fuente: Elaboración propia

También se constata una notable aceptación de los profesores con aspectos académicos, como el programa de acogida a los alumnos $(7,68)$, el contenido de las guías docentes $(8,00)$, las metodologías de enseñanza-aprendizaje $(7,62)$, las coordinación horizontes $(7,65)$ y verticales $(7,62)$ de las materias, la adecuación de los complementos formativos $(7,74)$, las tutorías $(7,85)$ y los criterios de evaluación $(7,53)$, con unos valores significativamente superiores a 7 .

Cuestiones como la organización del plan de estudios $(7,26)$ y la asignación de créditos a las distintas asignaturas $(7,32)$ y los programas de movilidad de los estudiantes $(7,26)$ obtienen unos valores significativamente superiores a 6 . Para concluir, el aspecto menos valorado es el procedimiento de asignación de los horarios $(6,21)$, que tan solo es significativamente mayor que 5. Por tanto, el profesorado parece bastante satisfecho con la nueva titulación de GADE, tanto en su contenido y estructura como en los métodos de enseñanzaaprendizaje utilizados, que permiten que un estudiante medio pueda conseguir los conocimientos y competencias definidos en su programa formativo.

\section{Recursos humanos y materiales}

Por lo que respecta a la opinión de los profesores sobre esta dimensión (ver tabla 4), destaca su alto grado de satisfacción con la materia que imparten $(8,32)$, la cualificación y experiencia de los profesores $(8,26)$ y los servicios para la docencia ofrecidos por los servicios de biblioteca, informática, etc. $(8,21)$, con valores significativamente superiores a 7 . También muestran ese mismo nivel de satisfacción en las cuestiones relacionadas con la cualificación y experiencia del personal de administración y servicios $(7,76)$, las instalaciones e infraestructuras $(7,79)$, los recursos tecnológicos $(7,82)$ y la información publicada en web del título $(7,68)$.

Un nivel de satisfacción significativamente superior a 6 se obtiene en cuestiones relacionadas con el sistema de garantía de la calidad $(7,24)$, las acciones de actualización docente $(6,97)$, el sistema de reclamaciones y sugerencias, y el Equipo Directivo del Centro $(7,50)$, en nuestro caso el Equipo Decanal de la Facultad de Ciencias Empresariales. Finalmente, el aspecto menos valorado es el plan de formación del profesorado $(6,38)$, que tan solo es significativamente mayor que 5.

Tabla 4. Estadísticos descriptivos sobre los recursos humanos y materiales 


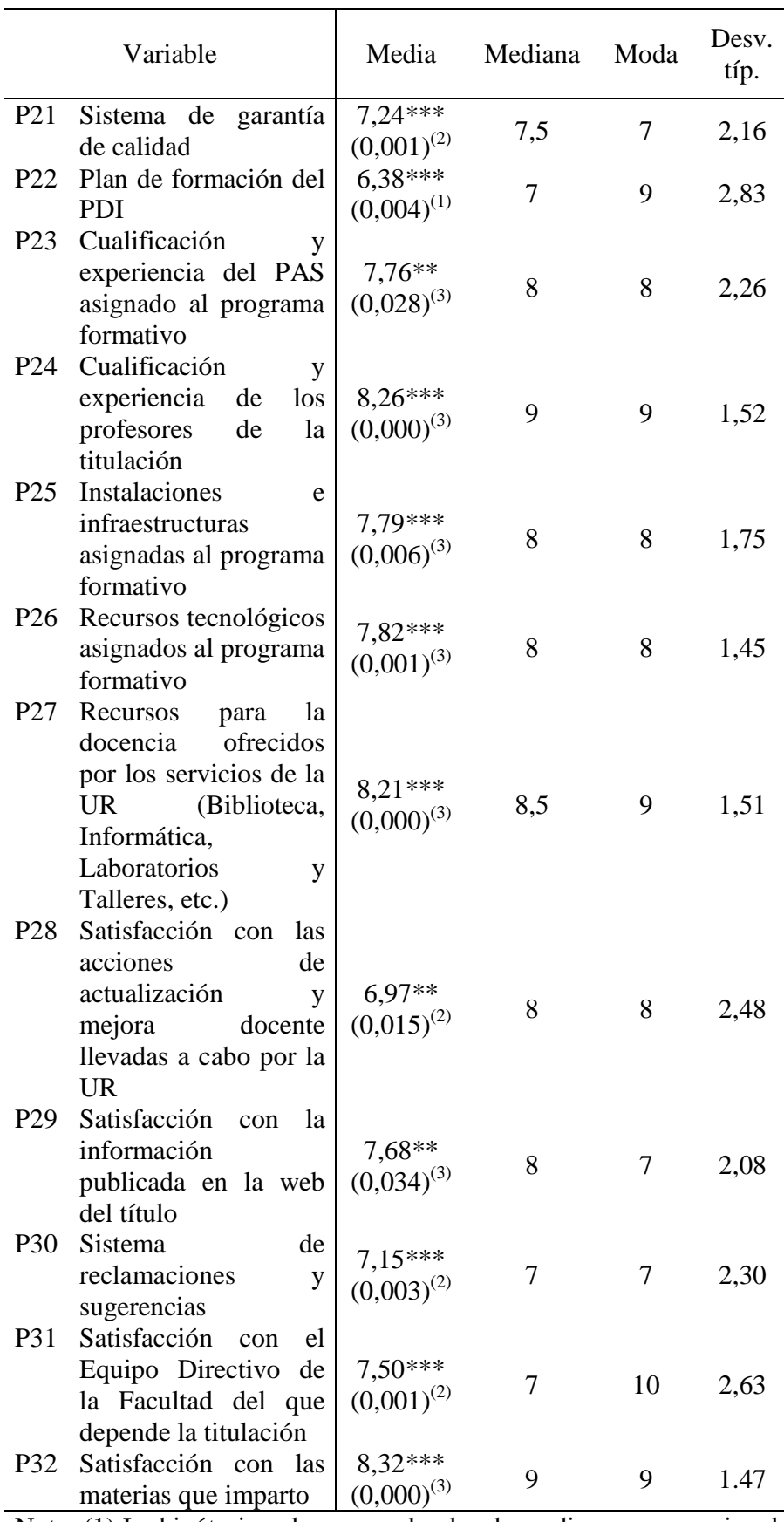

Nota: (1) La hipótesis nula es que el valor de media es menor o igual a 5, frente a la hipótesis alternativa de que es mayor a 5. (2) La hipótesis nula es que el valor de media es menor o igual a 6 , frente a la hipótesis alternativa de que es mayor a 6. (3) La hipótesis nula es que el valor de media es menor o igual a 7 , frente a la hipótesis alternativa de que es mayor a 7 . Los p-valor de la $t$-student, que permite contrastar dichas hipótesis, se recogen entre paréntesis. *** Significativo al 1\%. ** Significativo al 5\%. * Significativo al 10\%. Fuente: Elaboración propia

\section{CONCLUSIONES}

El objetivo del presente estudio ha sido evaluar el grado de satisfacción de los profesores con su experiencia docente en el Grado en Administración y Dirección de Empresas de la Universidad de La Rioja durante el curso académico 2015/16. Los resultados muestran, en general, un nivel aceptable de satisfacción por parte de los profesores con valores, para todos los ítems analizados, por encima de 5, que es el punto medio de la escala de medida utilizada, salvo el ítem de la dedicación de los estudiantes a lo planificado en la titulación.

De todas las dimensiones analizadas la correspondiente a "alumnado" es la de menor media global $(6,21) \mathrm{y}$, por tanto, donde hay más posibilidades de mejora. El profesorado se muestra escasamente satisfecho con el nivel de preparación con el que llegan los estudiantes a la Universidad y, una vez dentro de esta, con su nivel de asistencia a clase. Sin embargo, el aspecto que requiere una mayor atención es la dedicación del estudiante a sus estudios. Quizás una mejor definición del perfil de ingreso al Grado en Administración y Dirección de Empresas podría ayudar a resolver el primer problema detectado. Por otro lado, habría que diseñar algún procedimiento de incentivos para favorecer la asistencia a clase del alumno, así como un mayor compromiso y dedicación por parte de este hacia sus estudios.

Dentro de la dimensión "organización y desarrollo de la enseñanza”, con una valoración media global de 7,59 puntos, cabe señalar la excelente valoración que reciben las prácticas externas curriculares, que se desarrollan durante el segundo semestre del $4^{\circ}$ curso. Las prácticas externas en los planes de estudio vienen a reforzar el compromiso con la empleabilidad y a enriquecer la formación de los estudiantes en un entorno más acorde con la realidad diaria del ámbito profesional en el que desarrollarán su actividad futura. En el extremo opuesto, que requiere una actuación de mejora, está el procedimiento de asignación de horarios, que quizá debiera contemplar algún criterio que favoreciese la conciliación de la vida familiar y profesional de los profesores.

Los profesores universitarios también muestran un nivel de satisfacción elevado con la dimensión de "recursos humanos y materiales”, con una valoración media global de 7,59. Los aspectos mejor valorados son los relativos al personal, tanto de administración y servicios como a sus propios compañeros. En general, el profesor universitario está muy satisfecho con las asignaturas que imparte $(8,32)$, quizás debido al reducido tamaño de los grupos de la Universidad de La Rioja, y con los recursos materiales empleados en el proceso de enseñanzaaprendizaje, que presentan unos valores superiores a 7. Sin embargo, una cuestión a mejorar son los planes de formación del profesorado, que quizá debieran adaptarse más a sus necesidades formativas.

En conclusión, el profesorado universitario del Grado en Administración y Dirección de Empresas se muestra, en general, muy satisfecho con el nuevo plan de estudio adaptado al EEES y tan solo requieren de medidas de mejora los aspectos relativos al nivel de preparación con el que llegan los estudiantes a la Universidad, su asistencia a clase y dedicación a los estudios. También requieren de mejora la asignación de horarios entre los profesores y el plan de formación del profesorado.

\section{AGRADECIMIENTOS}

Este trabajo ha recibido apoyo financiero de la convocatoria de proyectos de innovación docente 2016/17 de la Universidad de La Rioja (PDI 8652).

\section{REFERENCIAS}


Alves, H. y Raposo, M. (2007). Conceptual model of student satisfaction in higher education. Total Quality Management, 18(5), pp. 571-588.

Anaya, D. y Suárez, J.M. (2007). Satisfacción laboral de los profesores de Educación Infantil, Primaria y Secundaria. Un estudio de ámbito nacional. Revista de Educación 344, pp. 217-243.

Ariza, T., Quevedo-Blasco, R. y Buela-Casal, G. (2014). Satisfaction of Social and Legal Sciences teachers with the introduction of the European Higher Education Area. European Journal of Psychology Applied to Legal Context 6, pp. 9-16.

Ariza, T., Quevedo-Blasco, R., Ramiro, M.T. y Bermúdez, M.P. (2013). Satisfaction of Health Science teachers with the convergence process of the European Higher Education Area. International Journal of Clinical and Health Psychology 3, pp. 197-206.

Athiyama, A. (1997). Linking student satisfaction and service quality perceptions: The case of university education. European Journal of Marketing, 31(7), pp. 528-540.

Bengoetxea, E. y Buela-Casal, G. (2013). The new multidimensional and user-driven higher education ranking concept of the European Union. International Journal of Clinical and Health Psychology, 13, pp. 6773.

Capelleras, J.L. y Veciana, J.M. (2001). Factores condicionantes de la calidad de la enseñanza universitaria. Barcelona: Universitat Autónoma de Barcelona.

Cardona, A., Barrenetxea, M.; Mijangos, J.J. y Olaskoaga, J. (2009). Concepto y determinantes de la calidad de la educación superior. Un sondeo de opinión entre profesores de universidades españolas. Archivos Analíticos de Políticas Educativas, 17, pp. 1-25.

Castro, A., Guillén, A., Quevedo, R., Bermúdez, M.P. y Buela, G. (2012). Doctoral Schools in Spain: Suggestions of Professors for their Implementation. Revista de Psicodidáctica, 17, pp. 199-217.

Comisión Europea (2009). Comunicado de Lovaina: El Proceso de Bolonia 2020 - El Espacio Europeo de Educación Superior en la nueva década. Comunicado de la Conferencia de Ministros europeos responsables de educación superior, Lovaina, 28-29 de abril de 2009.

Fernández, M.J., Carballo, R. y Galán, A. (2010). Faculty attitudes and training needs to respond the new European Higher Education challenges. Higher Education, 60, pp. 101-118.

Ferrer i Julià, F. (Dir.) (2004). Las opiniones y actitudes del profesorado universitario delante del Espacio Europeo de Educación Superior: Propuestas para la implementación del sistema de créditos europeos. Madrid: Ministerio de Educación y Ciencia.

Firdaus, A. (2006). The development of HEdPERF: a new measuring instrument of service quality for the higher education sector. International Journal of Consumer Studies, 30(6), pp. 569-581.
Fraile, A., López, V., Castejón, J. y Romero, R. (2013). La evaluación formativa en docencia universitaria y el rendimiento académico del alumnado. Aula Abierta, 41, pp. 23-34.

González, M.A. y Sanchís, C. (2014). Satisfacción de los egresados con la formación recibida en el Máster de Estudios Avanzados en Dirección de Empresas. Revista de Educación en Contabilidad, Finanzas y Administración de Empresas, 5, pp. 33-48.

Ion, G. y Cano, E. (2012). La formación del profesorado universitario para la implementación de la evaluación por competencias. Educación XX1, 15, pp. 249-270.

Ley Orgánica 6/2001, de 21 de diciembre, de Universidades. BOE 307, 24 de diciembre de 2001.

Ley Orgánica 4/2007, de 12 de abril, por la que se modifica la Ley Orgánica 6/2001, de 21de diciembre, de Universidades. BOE 89, 13 de abril de 2007.

Marcet, X. (2001). La captación de estudiantes en las universidades españolas: entre la inercia y el Marketing. Barcelona: La Demanda y la Captación de Estudiantes en las Universidades.

Mateo, J. y Vlachopoulos, D. (2013). Reflexiones en torno al aprendizaje y a la evaluación en la universidad en el contexto de un nuevo paradigma para la educación superior. Educación XX1, 16, pp. 183-208.

Ministerio de Educación (2010). Plan de acción 2010-2011. Objetivos de la educación para la década 2010-2020. Informe del consejo de ministros del 25 junio de 2010.

Monereo, C. (2010a). La formación del profesorado: Una pauta para el análisis e intervención a través de incidentes críticos. Revista Latinoamericana de Educación 52, pp. 149-178.

Monereo, C. (2010b). ¡Saquen el libro de texto! Resistencia, obstáculos y alternativas en la formación de los docentes para el cambio educativo. Revista de Educación 352, pp. 583-597.

Oldfield, B.M. y Baron, S. (2000). Student perceptions of service quality in a UK university business and management faculty. Quality Assurance in Education, 8, pp. 85-95.

Otero, C., Ferro, C. y Vila, M. (2012). Satisfacción del alumnado ante la implantación del modelo de EEES. Análisis comparativo. Revista Educativa Hekademos, 12, pp. 35-41.

Pérez, J.A., Lozano, J.A., Gómez, M. y Aguilera, A. (2010). Diseño de un instrumento para la evaluación de la satisfacción de la formación recibida de las diferentes asignaturas correspondientes al plan de estudios del grado en psicología de la Universidad de Sevilla. Revista de Enseñanza Universitaria, 36, pp. 45-61

Quevedo, R., Ariza, T. y Buela, G. (2015). Evaluación de la satisfacción del profesorado de ciencias con la adaptación al Espacio Europeo de Educación Superior. Educación XX1, 15(1), pp. 45-70 